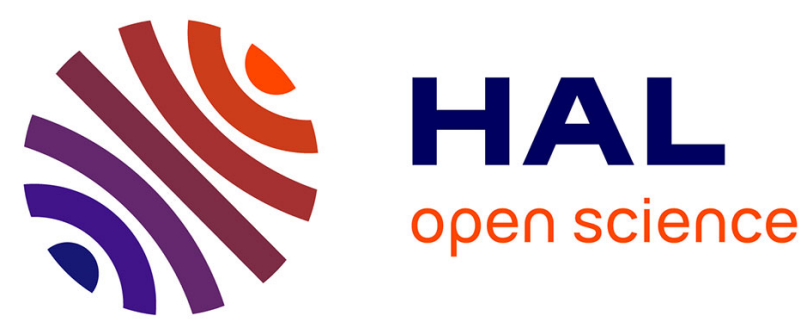

\title{
The Repertoire of Newly Developing Regulatory T Cells in the Type 1 Diabetes-Prone NOD Mouse Is Very Diverse
}

\author{
Ariel Galindo-Albarrán, Sarah Castan, Jérémy C Santamaria, Olivier Joffre, \\ Bart Haegeman, Paola Romagnoli, Joost P M van Meerwijk
}

\section{To cite this version:}

Ariel Galindo-Albarrán, Sarah Castan, Jérémy C Santamaria, Olivier Joffre, Bart Haegeman, et al.. The Repertoire of Newly Developing Regulatory T Cells in the Type 1 Diabetes-Prone NOD Mouse Is Very Diverse. Diabetes, 2021, 70 (8), pp.1729-1737. 10.2337/db20-1072 . hal-03375501

\author{
HAL Id: hal-03375501 \\ https://hal.science/hal-03375501
}

Submitted on 12 Oct 2021

HAL is a multi-disciplinary open access archive for the deposit and dissemination of scientific research documents, whether they are published or not. The documents may come from teaching and research institutions in France or abroad, or from public or private research centers.
L'archive ouverte pluridisciplinaire HAL, est destinée au dépôt et à la diffusion de documents scientifiques de niveau recherche, publiés ou non, émanant des établissements d'enseignement et de recherche français ou étrangers, des laboratoires publics ou privés. 
The repertoire of newly developing regulatory $T$ cells in the type I diabetes-prone NOD mouse is very diverse Ariel Galindo-Albarrán ${ }^{1,2}$, Sarah Castan ${ }^{1}$, Jérémy C. Santamaria ${ }^{1}$, Olivier P. Joffre ${ }^{1}$, Bart Haegeman², Paola Romagnoli ${ }^{1}$, and Joost P.M. van Meerwijk ${ }^{1,3}$

\footnotetext{
${ }^{1}$ Toulouse Institute for Infectious and Inflammatory Diseases (Infinity), INSERM UMR1291 CNRS UMR5051 - University Toulouse III, Toulouse, France

${ }^{2}$ Station d'Écologie Théorique et Expérimentale, CNRS, Moulis, France; Université Paul Sabatier, Moulis, France

${ }^{3}$ corresponding author
}

Key words: $\quad$ Autoimmune disease, type I diabetes, regulatory T lymphocytes, thymus, repertoire, $\mathrm{T}$ cell receptors for antigen, mouse Abbreviations: $\quad$ NOD, non-obese diabetic; TCR, T cell receptor for antigen; Treg, regulatory T lymphocyte; CDR3, complementarity determining region 3;

Running title: $\quad$ TCR-diversity of newly developed NOD Treg

Corresponding author: Prof. Joost P.M. van Meerwijk

Toulouse Institute for Infectious and Inflammatory Diseases, Toulouse, France

Phone $\quad+33562748381$

E-mail_Joost.van-Meerwijk@inserm.fr 


\section{Summary}

Regulatory T lymphocytes expressing the forkhead/winged helix transcription factor Foxp3 (Treg) play a vital role in the protection of the organism from autoimmune disease and other immunopathologies. The antigen-specificity of Treg plays an important role in their in vivo activity. We therefore assessed the diversity of the T cell receptors for antigen (TCR) expressed by Treg newly developed in the thymus of autoimmune type I diabetes-prone NOD mice and compared it to the control mouse strain C57BL/6. Our results demonstrate that usage of the TCR $\alpha$ and TCR $\beta$ variable (V) and joining (J) segments, length of the complementarity determining region (CDR) 3, and the diversity of the TCR $\alpha$ and TCR $\beta$ chains are comparable between NOD and C57BL/6 mice. Genetic defects affecting the diversity of the TCR expressed by newly developed Treg therefore do not appear to be involved in the etiology of type I diabetes in the NOD mouse. 


\section{Introduction}

Regulatory T lymphocytes expressing the forkhead/winged helix transcription factor Foxp3 (Treg) play a major and even vital role in the control of innate and adaptive immunity and are also involved in tissue repair (1-3). Using a large variety of suppressor-effector mechanisms, Treg inhibit immunity and thus avoid autoimmune pathology, chronic inflammation, and rejection of the semi-allogeneic fetus (2). The vital character of immune-regulation by Treg is best illustrated by the rapidly lethal autoimmune and inflammatory syndromes that develop in humans and mice carrying loss-of-function mutations in the locus encoding the master regulator of Treg-development and function, FOXP3/Foxp3 $(4,5)$. More subtle defects in Treg may be involved in the etiology of autoimmune pathology (6).

Treg can differentiate from T cell-precursors during $\mathrm{T}$ cell-development in the thymus and from mature conventional T lymphocytes during immune-responses in the periphery (2). In the thymus, Treg development requires high affinity interactions of the $T$ cell receptor for antigen (TCR) expressed by T cell-precursors with $\mathrm{MHC}$ /peptide complexes expressed mainly by medullary thymic stromal cells (7). Since the latter cells present peptides derived from tissue-antigens, in part through the action of the transcription factor Autolmmune REgulator (AIRE)(8), an autospecific Treg repertoire thus develops $(9,10)$. Treg development in the thymus also requires cytokines such as IL-2 and IL-15 (11) as well as signals through costimulatory receptors (12-14).

Defects in Treg-development are thought to be involved in the etiology of autoimmune pathology (6). Thus, the very aggressive and rapidly lethal autoimmune syndrome developing in AIRE-deficient Non-Obese Diabetic (NOD) mice appears, at least in part, due to defective selection of the Treg repertoire early in life (15). Also mice deficient in IL-2 or its receptor rapidly succumb to autoimmune pathology, but the respective roles herein of defective 
intrathymic development and peripheral survival and function of Treg remain to be assessed (11). NOD mice deficient in CD28, in which substantially less Treg develop in the thymus, also develop more aggressive type I diabetes (16).

It has been suggested that the TCR-repertoire expressed by Treg developing in the thymus of type I diabetes (T1D)-prone NOD mice is less diverse than that developing in the thymus of C57BL/6 (B6) mice, frequently used as a T1D-resistant reference (17-19). The results were based on analysis of $\mathrm{CD} 25$-expressing $\mathrm{CD} 4^{+} \mathrm{CD} 8^{-}$thymocytes, presumably developing Treg. However, our laboratory recently demonstrated that, in adult mice, actually only a fraction of thymic Treg are cells that had recently developed, the rest being cells that had recirculated from the periphery back to the thymus (20). Moreover, we recently demonstrated that the NOD thymus contains a particularly high proportion of recirculating Treg (21). Also, CD25 is not only expressed by Treg but also by immature precursors (22). To assess the potential involvement, in the etiology of type I diabetes in the NOD mouse, of genetic defects affecting the diversity of the TCRs expressed by newly developed Treg, we therefore readdressed this issue using mutant NOD and B6 mice in which newly developed Treg can be distinguished from recirculating cells, and in which Treg can be isolated based on Foxp3-expression. In Rag2-Gfp mice, the Rag2-promoter drives expression of Green Fluorescent Protein (GFP)(23). Transcription of the GFP-encoding sequence thus terminates at positive selection at the CD4/CD8 double positive stage of T cell development and the accumulated GFP degrades with a half-life of 56 hours $(24,25)$. In the thymus, newly developed Treg retain sufficient green fluorescence to distinguish them from recirculating cells that had entirely lost fluorescence (20). In Foxp3-Thy $1^{a}$ mice, the allelic cell-surface reporter Thy1.1 is expressed under control of the Foxp3-promoter allowing detection of Foxp3-expressing Treg without fixation/permeabilization procedures, thus allowing for isolation of mRNA required for TCR 
repertoire analysis by high throughput mRNA-sequencing. Using Rag2-Gfp Foxp3-Thy $1^{a}$ mice, we here firmly demonstrate that the TCR-repertoire expressed by Treg newly developed in the thymus of the T1D-prone NOD mouse is as diverse as that developing in the resistant mouse strain B6. 


\section{Research design and methods}

Mice

B6 mice expressing the Foxp3-Thy $1^{a}$ mutation and a Rag2-Gfp transgene were previously described (26). The two mutations were introduced into the NOD genetic background by speed-backcrossing (27). After all Idd-loci were of NOD allotype (six generations), the mice were crossed to NOD mice for another ten generations. Wt NOD.CD45.2 and B6.CD45.1 congenic mice were bred in our colony.

\section{Flow-cytometry}

Thymocytes from female eight-week-old wt NOD and B6 mice were incubated with antiFc $\gamma$ RIII/Fc $\gamma$ RII antibody (2.4G2) and then labelled with EF450-labelled anti-CD8 $\beta$ (H35-17.2), PE-labelled anti-CCR7 (4B12)(both from eBioscience), APC-Cy7-labelled anti-CD4 (GK1.5), PECy7-labelled CD25 (PC61)(both from BD Biosciences), and BV605-labelled anti-CD73 (TY/11.8 Biolegend). Thus labelled cells were stained with a TCR V $\beta$ screening FITC-labeled antibodypanel (BD Biosciences). Cells were then stained with EF660-labelled anti-Foxp3 (FJK-16s, eBioscience) according to the manufacturer's instructions. Thus labelled cells were analyzed using a Fortessa flow cytometer (BD Biosciences) and data analyzed using FlowJo software (Tree Star).

TCR-sequencing

Pooled thymocytes from eight to ten, eight-week-old female Foxp3-Thy $1^{a}$ Rag2-Gfp NOD and $\mathrm{B} 6$ mice were complement-depleted of $\mathrm{CD} 8^{+}$cells using an anti-CD8 $\alpha$ hybridoma (31M) supernatant. Remaining cells were stained with PE-labelled anti-CD4 (GK1.5), EF450-labelled anti-CD8 $\beta$ (H35-17.2)(both from eBiosciences), and APC-labelled anti-Thy1.1 (OX7, BD 
Biosciences). $10^{5} \mathrm{CD}^{+} \mathrm{CD} 8^{-}$Thy $1.1^{+} \mathrm{GFP}^{+}$Treg were sorted using the FACS-ARIA-II cell sorter (BD Biosciences). TCRseq was performed as previously described, with minor modifications $(28,29)$. mRNA was extracted by Nucleospin RNA XS (Macherey-Nagel) according to the manufacturer's instructions and was quality controlled (RIN > 8) using Agilent 2100 BioAnalyzer. cDNA was prepared as follows. mRNA was reverse transcribed using primers aligning to the sequences encoding the constant regions of TCR $\alpha$ (CTCAGCGTCATGAGCAGGTTAAAT; CAGGAGGATTCGGAGTCCCATAA; TTTTACAACATTCTCCAAGA; TTCTGAATCACCTTTAATGA; ATGAGATAATTTCTACACCT; TTTGGCTTGAAGAAGGAGCG; TTCAAAGCTTTTCTCAGTCA; TGGTCTCTTTGAAGATATCT) and TCRß (GGTAGCCTTTTGTTTGTTTG; CCCCTGGCCAAGCACACGAG; TGCCATTCACCCACCAGCTC; GCTATAATTGCTCTCCTTGT; TTGCGAGGATTGTGCCAGAA; CTTGTCCTCCTCTGAAAGCC; GCCTCTGCACTGATGTTCTG). 5' adapters containing unique molecular identifiers (UMI) were added using a template-switch reaction (TACACGACGCTCTTCCGATCUNNNNUNNNNUNNNNUCTTrGrGrGrGrG). Libraries were prepared as follows. The first PCR reaction was performed with $5^{\prime}$ primer TACACGACGCTCTTCCGATC and 3' primers AAGTCGGTGAACAGGCAGAG for Tcra and TGATGGCTCAAACAAGGAGACC for Tcrb $\left(2^{\prime} 95^{\circ} \mathrm{C}, 10\right.$ cycles of $20^{\prime \prime} 95^{\circ} \mathrm{C}, 15^{\prime \prime} 59^{\circ} \mathrm{C}, 45^{\prime \prime} 70^{\circ} \mathrm{C}$, final incubation $3.5^{\prime} 70^{\circ} \mathrm{C}$ ). The amplicons were then purified using Agenecourt AMPure XP beads. The second, semi-nested PCR was performed with 5' primer TACACGACGCTCTTCCGATC and 3' primers AGCAGGTTCTGGGTTCTGGA for Tcra and GGGTGGAGTCACATTTCTCAGAT for Tcrb (2' $95^{\circ} \mathrm{C}$; 20 cycles of $20^{\prime \prime} 95^{\circ} \mathrm{C}, 15^{\prime \prime} 59^{\circ} \mathrm{C}, 45^{\prime \prime} 70^{\circ} \mathrm{C}$, final incubation $3.5^{\prime} 70^{\circ} \mathrm{C}$ ). The third PCR was performed using $5^{\prime}$ primer AATGATACGGCGACCACCGAGATCTACACTCTTTCCCTACACGACGCTCTTCCGATC and 3' primers CAAGCAGAAGACGGCATACGAGATXXXXXXXXGTGACTGGAGTTCAGACGTGTGCTCTTCCGATCTA 
GCAGGTTCTGGGTTCTGGA for Tcra and

CAAGCAGAAGACGGCATACGAGATXXXXXXXXGTGACTGGAGTTCAGACGTGTGCTC

TTCCGATCTGGGTGGAGTCACATTTCTCAGAT for Tcrb (in which XXXXXXXX is a sequencing index) $\left(2^{\prime} 95^{\circ} \mathrm{C} ; 1\right.$ cycle of $20^{\prime \prime} 95^{\circ} \mathrm{C}, 15^{\prime \prime} 59^{\circ} \mathrm{C}, 45^{\prime \prime} 70^{\circ} \mathrm{C}, 5$ cycles of $20^{\prime \prime} 95^{\circ} \mathrm{C}, 15^{\prime \prime} 75^{\circ} \mathrm{C}, 45^{\prime \prime}$ $70^{\circ} \mathrm{C}$, final incubation $3.5^{\prime} 70^{\circ} \mathrm{C}$ ). For the fourth PCR, 5' primer AATGATACGGCGACCACCGA and 3' primer CAAGCAGAAGACGGCATACGA were used $\left(2^{\prime} 95^{\circ} \mathrm{C}, 5\right.$ cycles of $20^{\prime \prime} 95^{\circ} \mathrm{C}, 15^{\prime \prime}$ $60^{\circ} \mathrm{C}, 45^{\prime \prime} 70^{\circ} \mathrm{C}$; final incubation of $3.5^{\prime}$ at $70^{\circ} \mathrm{C}$ ). The quality of each library was verified using Agilent 2100 BioAnalyzer (mean peak size 640 bp). The samples were indexed and sequenced with 300pb paired-end on a MiSeq sequencer (Illumina). Thus, three Tcra and three Tcrb libraries per mouse-strain were generated.

\section{Processing of TCRseq data}

The reads were preprocessed with the toolkit pRESTO (30) as follows. Using FilterSeq, reads with a quality $>20$ were selected. Using MaskPrimers and PairSeq algorithms, the sequences corresponding to the TCR $\alpha$ constant region (AGCAGGTTCTGGGTTCTGGA) or TCR $\beta$ constant region (GGGTGGAGTCACATTTCTCAGAT) and indicating location of the UMI (CTTGGGGG) were searched for and indexed to the head of the paired reads. Using BuildConsensus, consensus-sequences of the reads with the same UMI were constructed. Next, the forward and reverse reads were aligned to assemble the Tcra and the Tcrb sequences (AssemblePairs) and the UMI groups containing at least two reads were selected. The sequenced fragments from each selected UMI were aligned to the Tcra or Tcrb genomic region using the toolkit MiXCR (31) with the tools "align" and "assemble". The aligned fragments were exported as "clonotype-tables" using the tool "exportClones". 
Using VDJtools (32) and customized R-scripts these clonotype-tables were then processed and graphs generated. The V-J-usage plots were generated using PlotFancyVJUsage command. The CDR3 lengths were calculated based on the information in the clonotypetable. The numbers of " $\mathrm{P}$ " and " $\mathrm{N}$ " nucleotides were extracted from the clonotype-tables using the command "exportClones VGeneWithP". Rarefaction plots and diversity statistics were calculated and graphed using the R package "iNEXT". We used three different diversity measures: Chao1 estimate, Shannon diversity and Simpson diversity. All three provide an estimate of the number of clonotypes in the repertoire, but differ in the extent to which they discount rare clonotypes. The more rare and therefore difficult to detect clonotypes are discounted, the more precisely the diversity measure can be estimated. By jointly considering these three diversity measures, we cover the full diversity information contained in the sample data (33). All custom scripts used are available at: https://github.com/arielgalindoalbarran/NOD_TCR_repertoire.git.

\section{Data and Resource Availability}

TCRseq data generated during the current study are available in the Gene Expression Omnibus (GEO) database (http://www.ncbi.nlm.nih.gov/gds) under accession number GSE159001. The other datasets are available from the corresponding author upon reasonable request. The mutant mice used in the current study are available from the corresponding author upon reasonable request 


\section{Results}

Only a limited proportion of Treg in the thymus of adult NOD mice are newly developed cells We and others previously showed that the pool of Treg in the thymus is constituted of newly developed and of fully mature cells. The latter Treg are mostly cells that had recirculated from the periphery back to the thymus. We therefore analyzed NOD mice carrying a Rag2-Gfp transgene and the Foxp3-Thy $1^{a}$ mutation in which we distinguished newly developed GFP' from recirculating GFP', surface Thy1.1+ Treg (20). Using flowcytometry, we found that $32 \pm 11 \%$ of thymic $\mathrm{CD}^{+}{ }^{+} \mathrm{CD} 8$-Foxp3 ${ }^{+}$Treg were $\mathrm{GFP}^{+}$in eight-weekold NOD females (Fig. 1A). This extends our previous finding that in the thymus of adult B6 mice only a minority of Treg are newly developed cells $(20,21)$. In this study we focused our attention on these newly developed Treg.

The $V$ and J segment-use in TCR $\alpha$ - and TCR $\beta$-chains expressed by newly developed NOD and B6 Treg is is very similar and comparably diverse To assess the diversity of TCR expressed by newly developed Treg, we first analyzed their TRBV (i.e. TCR- $V_{\beta}$ ) repertoire by flow-cytometry. The commercially available anti-TCR- $V_{\beta}$ antibody panels are FITC-labeled, which is incompatible with the GFP-based identification of newly developed Treg in Rag2-Gfp transgenic mice. We therefore identified newly developed nuclear Foxp3 ${ }^{+}$Treg in wt mice using their $\mathrm{CCR} 7^{\text {high }} \mathrm{CD} 73^{-/ \text {low }}$ phenotype that we recently described (21)(Fig. S1). Using antibodies to 15 distinct TRBV, we found that the TRBV repertoires expressed by newly developed NOD and B6 Treg were distinct (Fig. S2A), but similarly diverse, even if the very small difference in the diversities (3.5\%) was statistically significant (Fig. 1B). 
To study the TRAV repertoire expressed by newly developed Treg, we sorted

$\mathrm{CD}^{+}{ }^{+} \mathrm{CD} 8-$ Thy $1.1^{+} \mathrm{GFP}^{+}$thymocytes from Foxp3-Thy $1^{a}$ Rag2-Gfp NOD and B6 mice, isolated mRNA, and then performed high throughput sequencing of Tcra mRNA ("TCRseq"). We used unique molecular identifiers (UMI) to identify sequences derived from individual mRNAs. Whereas we observed substantial differences between the TRAV-repertoires of NOD and B6 Treg, they were similarly diverse (Fig. 2A and table S1). Using TCRseq we also confirmed the similar diversities of the TRBV repertoires expressed by newly developed NOD and B6 Treg (Fig. 2B, table S2). The diversities (i.e. D-values, Shannon diversity) of the TRBV-repertoires as determined by TCRseq were higher than those determined by flow-cytometry analyses (cf. Figs. 2B vs. 1B) because, whereas existing antibodies do not cover the entire range of TRBV-segments, TCRseq detects all of them. Comparison of the TRBV-repertoires as determined by flow-cytometry vs. TCRseq revealed, as expected, substantial though imperfect similarity (Fig. S3). Differences are probably due to the facts that the two techniques analyze individual cells and mRNAs, respectively, and that each TRBV has its own promoter region, potentially leading to distinct levels of mRNAs in individual cells.

The V-J combination used in a TCR is a critical parameter in its specificity. We therefore assessed the distinct V-J combinations used in TCR expressed by newly developed Treg in NOD and B6 mice. We found some substantial differences in V-J associations used in TCR $\alpha$ and TCR $\beta$ expressed by newly developed NOD vs. B6 Treg (Table S3, S4), but the diversities of these associations appeared very large and comparable (Fig. 2C, D). Rearrangements of the Tcra locus continue until positive selection occurs thus, with time, using more-upstream V and more-downstream J segments (24). To assess the "stringency" of positive selection, we therefore assessed the position-weighted usage of $\mathrm{V}$ and $\mathrm{J}$-segments and found no statistically significant differences between the NOD and B6 Tcra-sequences (Fig. 2C, S4). 
The composition of the CDR3 of TCRs expressed by newly developed NOD and B6 Treg is similar

During the course of the distinct thymic selection events, the CDR3s of TCR $\alpha$ and TCR $\beta$ chains gradually shorten (34-37). To assess if newly developed Treg have undergone more or less stringent selection In NOD vs. B6 mice, we therefore next assessed, using the TCRseq data, the CDR3-lengths of the TCR $\alpha$ and TCR $\beta$ chains. The average CDR3-lengths of the TCR $\alpha$ and TCR $\beta$-chains were identical between NOD and B6 mice (Fig. 2E, F, left panels). Also the CDR3-length-distributions were practically identical for Treg newly developed in NOD and B6 mice, even if we observed some very small but statistically significant differences (Fig. 2E, $\mathrm{F}$, right panels).

Non-germline-encoded CDR3 amino-acids appear to reduce the affinity of the TCR's interactions with $\mathrm{MHC}$ molecules (38). These amino-acids are mostly encoded by $\mathrm{N}$ and $\mathrm{P}$ nucleotides. To assess this parameter, we extracted from the TCRseq data the average numbers and distributions of $\mathrm{N}$ and of $\mathrm{P}$ nucleotides in the CDR3 regions of the TCR $\alpha$ and TCR $\beta$ chains expressed by newly developed Treg. We found that they were practically identical in NOD and B6 mice, even if we observed some very small but statistically significant differences (Fig. 2G-J).

The diversity of TCR $\alpha$ and TCR $\beta$ chains expressed by newly developed NOD and B6 Treg is similar

We next assessed the total diversity of the TCR $\alpha$ and TCR $\beta$ "clonotypes" (as characterized by V-segment-usage, CDR3-sequence, and J-segment-usage) expressed by newly developed Treg in NOD and B6 thymi. Rarefaction plots showed similar diversities of TCR $\alpha$ and TCR $\beta$ 
clonotypes expressed by NOD and B6 Treg (Fig. 3A, S5A). Chao1, Shannon, and Simpson indexes confirmed the similar diversities of TCR $\alpha$ and TCR $\beta$ clonotypes in NOD and B6 samples (Fig. 3B, S5B).

Previous reports indicated that, as compared to thymic Treg in B6 mice, those in NOD mice expressed a limited diversity of TCR $\alpha$ chains containing TRAV12 and TRAV9 (TRAV2 and TRAV17, respectively, according to the nomenclature of the international ImMunoGeneTics information system, www.imgt.org) $(17,18)$ We therefore also separately analyzed the diversity of TCR $\alpha$ clonotypes using these TRAV. As shown in Fig. 3C and D and S5C, we observed similar diversities in NOD and B6 samples.

The composition and diversity of TCR $\alpha$ and TCR $\beta$ chains expressed by recirculating Treg in NOD and B6 thymi are similar

These data indicate that the diversities of TCRs expressed by Treg newly developed in the NOD and B6 thymi are similar. This conclusion disagrees, in part, with earlier reports $(17,18)$. One of the several potentially underlying reasons is that, in contrast to the earlier studies, we studied newly developed cells. A difference in the diversities of the very numerous recirculating cells in the pools of Treg in NOD vs. B6 thymi (i.e. GFP- cells in Fig. 1A) might provide a partial explanation. To assess this possibility, we performed TCRseq analyses on recirculating $\mathrm{CD}^{+} \mathrm{CD}^{-}{ }^{-}$Thy $1.1^{+} \mathrm{GFP}^{-}$thymocytes from Foxp3-Thy $1^{a}$ Rag2-Gfp NOD and B6 mice. Recirculating NOD vs. B6 Treg expressed similarly (though not identically) diverse TRAV and TRBV repertoires (Fig. S6A, B) and V-J associations (Fig. S6C, D), and they had similar distributions and average values for CDR3-lengths and numbers of $\mathrm{N}$ and $\mathrm{P}$ nucleotides (Fig. S6E-J). They also expressed similarly diverse TCR $\alpha$ and TCR $\beta$ repertoires 
(Fig. S7A, B). Finally, also the TRAV2 and TRAV17 repertoires expressed by recirculating Treg were similarly diverse in NOD vs. B6 thymi (Fig. S7C, D). 


\section{Discussion}

The data we presented here indicate that the CDR3-characteristics and the diversities of the TCR $\alpha$ and TCR $\beta$ repertoires expressed by Treg newly developed in the thymus of the type I diabetes-prone NOD mouse are very similar to those found in the autoimmune-diseaseresistant mouse-strain B6. This conclusion is principally based on our deep TCR-sequencing analysis of Tcra and Tcrb-derived mRNAs that allowed for an unbiased analysis of thousands of cells.

Importantly, TCRseq analysis requires numbers of cells that are very difficult to obtain from single mice. We therefore pooled eight to ten mice per sample analyzed. Whereas pooling several mice would have reinforced the statistical significance of differences found, it eliminates potential inter-individual differences which may be biologically significant. It would therefore be of substantial interest to measure the inter-individual variation of the diversities and the private and public TCR-repertoires expressed by newly developed cells. To address such questions, the TCRseq procedure will need to be further substantially refined.

Despite the generally high levels of similarity, we observed some small differences between the TCR-repertoires expressed by Treg newly developed in thymi of NOD vs. B6 mice. Thus, whereas they were similarly diverse, the TRBV and TRAV usages were distinct. This result was most probably due to the different alleles of MHC class II molecules expressed by the two mouse strains, $\mathrm{I}-\mathrm{A}^{\mathrm{g} 7}$ for NOD and $\mathrm{I}-\mathrm{A}^{\mathrm{b}}$ for $\mathrm{B} 6$ and, for TRBV, to the distinct MMTV-encoded superantigens encoded in their genomes (39). We also observed some minor but statistically significant differences in CDR3 $\beta$-length distribution between TCRs expressed by newly developing Treg in NOD vs. B6 mice. Together with some minor differences in the numbers of $\mathrm{N}$ and $\mathrm{P}$ nucleotides, these observations may again be due to the distinct $\mathrm{I}-\mathrm{A}$ alleles expressed in NOD vs. B6 mice and/or to potential slightly lower stringencies of the positive and negative 
selection processes in NOD as compared to B6 mice (34-37). Analyses involving MHCcongenic mice will be required to shed light on these different possibilities.

Our results are in part different from previously published data $(17,18)$. In the latter studies, thymic Treg were defined as $\mathrm{CD} 4^{+} \mathrm{CD} 8{ }^{-} \mathrm{CD} 25^{+}$cells. This population is heterogeneous and contains, besides newly developed Treg, also immature Treg precursors and recirculating Treg $(20,40)$. The recirculating Treg population in the thymus grows steadily with age and is particularly prominent in NOD mice (21). In five- to six-week-old NOD and B6 mice, analyzed in the previous reports, this population represents $47 \pm 10 \%$ and $37 \pm 9 \%$ of thymic Treg, respectively (21). However, our analysis of the TCR-repertoires expressed by recirculating Treg indicated that they were similarly diverse in NOD vs. B6 mice, thus excluding this potentially confounding factor. Interestingly, we found that the diversity of the TCR expressed by recirculating Treg in the thymus is very close to that of newly developed cells. Since mainly activated Treg migrate back to the thymus (21), this observation suggests a massive activation of autospecific Treg in the periphery, consistent with previously published data (41). Moreover, approximately half of the previously analyzed $\mathrm{CD} 4^{+} \mathrm{CD} 8 \mathrm{CD} 25^{+}$cells are CD25 ${ }^{+}$Foxp3- Treg precursors (22). Thus, the newly developed Treg population actually represents a small minority of the cells previously analyzed. Using tools that have more recently become available, we were able to sort a pure population of newly developed Foxp3 ${ }^{+}$ Treg. Moreover, the protocol used previously to determine the TCR-V $\alpha$ encoding sequences was very different from ours. Whereas in the former case TRAV2 and TRAV17-targeted and non-UMI-based amplification of TRAV-CDNAs followed by cloning and sequencing was used, we used a non-biased and UMI-based protocol and high-throughput sequencing. The combined differences between the approaches taken previously and in the current study somehow explain the discrepancy of the results. Whatever the precise reason(s), our data 
indicate that the diversities of the TCR $\alpha$ and TCR $\beta$ repertoires expressed by newly developed Treg are very similar in NOD and B6 mice. Also when focusing our analysis on the two TRAVs analyzed previously, we found similar diversities between the repertoires expressed by newly developed NOD vs. B6 Treg.

We therefore conclude that the repertoire of TCRs expressed by newly developed Treg in the NOD mouse is of similar diversity as that expressed in the control B6 strain. Importantly, this does not exclude the possibility that essential antigenic specificities may be lacking in the NOD Treg repertoire. Thus, the presentation of tissue restricted antigens by medullary thymic stromal cells is known to be involved in shaping the TCR-repertoire expressed by Treg (15). Genetic differences in expression of tissue-restricted antigens, as previously reported, may for example be involved in the susceptibility of the NOD mouse to T1D (42). 


\section{Acknowledgements}

We are very grateful to the following persons for excellent technical assistance: Fatima L'Faqihi, Valérie Duplan-Eche, Anne-Laure Iscache, Lidia De la Fuente, Paul Menu of the CPTP Cytometry platform; Adrien Castinel and Gwenolah Annonay of the GeT-PlaGE Genotoul; and the personnel of the Inserm USO06 ANEXPLO/Crefre animal facility. The authors are grateful to the members of the 'T-cell mediated immune Tolerance team' and to Sylvie Guerder for discussion and input in the project. JPMvM is grateful to the staff of the Biochemistry Institute of the Lausanne University, Epalinges, Switzerland, for its hospitality. This work was financially supported by the Fondation pour la Recherche Médicale (to JVM, DEQ20160334920; to JCS, FDT201904008280); the IdEx Toulouse (to PR); the Région Midi Pyrénées (to JvM, 15/06/12.05); the Agence Nationale pour la Recherche (to PR, ANR-16-CE15-0015-01). AGA, SC, JS, OJ, and JVM dedicate this work to the memory of their late, much respected and regretted colleague PR.

\section{Conflicts of interest}

The authors declare that they do not have any conflicts of interest with the current study. 


\section{Author contributions}

A.G.A. designed and performed bio-informatic analysis and helped writing the manuscript;

S.C. designed and performed experiments and helped writing the manuscript; J.C.S. designed and performed experiments, performed analysis and helped writing the manuscript; O.P.J. helped designing the study and analyzing the data and reviewed the manuscript; B.H. analyzed data and helped writing the manuscript; P.R. helped designing the study and analyzing the data; J.P.M.v.M. designed and supervised the study, wrote the manuscript, and is the guarantor of this work. 


\section{References}

1. Sakaguchi S, Miyara M, Costantino CM, Hafler DA. FOXP3+ regulatory T cells in the human immune system. Nat Rev Immunol. 2010;10(7):490-500.

2. Josefowicz SZ, Lu L-FF, Rudensky AY. Regulatory T cells: mechanisms of differentiation and function. Annu Rev Immunol. 2012;30(1):531-64.

3. Panduro M, Benoist C, Mathis D. Tissue Tregs. Annu Rev Immunol. 2016;34(1):60933.

4. Bennett $\mathrm{CL}$, Christie J, Ramsdell F, Brunkow ME, Ferguson PJ, Whitesell L, et al. The immune dysregulation, polyendocrinopathy, enteropathy, $X$-linked syndrome (IPEX) is caused by mutations of FOXP3. Nat Genet. 2001;27(1):20-1.

5. Brunkow ME, Jeffery EW, Hjerrild KA, Paeper B, Clark LB, Yasayko SA, et al. Disruption of a new forkhead/winged-helix protein, scurfin, results in the fatal lymphoproliferative disorder of the scurfy mouse. Nat Genet. 2001;27(1):68-73.

6. Long SA, Buckner JH. CD4+FOXP3+ T Regulatory Cells in Human Autoimmunity: More Than a Numbers Game. J Immunol. 2011;187(5):2061-6.

7. Romagnoli P, van Meerwijk JPM. Thymic selection and lineage commitment of CD4(+)Foxp3(+) regulatory T lymphocytes. Prog Mol Biol Transl Sci. 2010;92:251-77.

8. Klein L, Kyewski B, Allen PM, Hogquist KA. Positive and negative selection of the T cell repertoire: what thymocytes see (and don't see). Nat Rev Immunol. 2014;14(6):37791.

9. Romagnoli P, Hudrisier D, van Meerwijk JPM. Preferential Recognition of Self Antigens Despite Normal Thymic Deletion of CD4+CD25+ Regulatory T Cells. J Immunol. 2002;168(4):1644-8. 
10. Hsieh CS, Liang Y, Tyznik AJ, Self SG, Liggitt D, Rudensky AY. Recognition of the peripheral self by naturally arising CD25+CD4+ T cell receptors. Immunity. 2004;21(2):267-77.

11. Apert C, Romagnoli P, van Meerwijk JPMJPM. IL-2 and IL-15 dependent thymic development of Foxp3-expressing regulatory T lymphocytes. Protein Cell. 2018;9(4):322-32.

12. Tai X, Cowan M, Feigenbaum L, Singer A. CD28 costimulation of developing thymocytes induces Foxp3 expression and regulatory T cell differentiation independently of interleukin 2. Nat Immunol. 2005;6(2):152-62.

13. Mahmud SA, Manlove LS, Schmitz HM, Xing Y, Wang Y, Owen DL, et al. Costimulation via the tumor-necrosis factor receptor superfamily couples TCR signal strength to the thymic differentiation of regulatory T cells. Nat Immunol. 2014;15(5):473-81.

14. Coquet JM, Ribot JC, Bąbała N, Middendorp S, van der Horst G, Xiao Y, et al. Epithelial and dendritic cells in the thymic medulla promote CD4+Foxp3+ regulatory $\mathrm{T}$ cell development via the CD27-CD70 pathway. J Exp Med. 2013;210(4):715-28.

15. Yang S, Fujikado N, Kolodin D, Benoist C, Mathis D. Regulatory T cells generated early in life play a distinct role in maintaining self-tolerance. Science. 2015;348(6234):58994.

16. Salomon B, Lenschow DJ, Rhee L, Ashourian N, Singh B, Sharpe A, et al. B7/CD28 costimulation is essential for the homeostasis of the $C D 4+C D 25+$ immunoregulatory $T$ cells that control autoimmune diabetes. Immunity. 2000;12(4):431-40.

17. Ferreira C, Singh Y, Furmanski AL, Wong FS, Garden OA, Dyson J. Non-obese diabetic mice select a low-diversity repertoire of natural regulatory T cells. Proc Natl Acad Sci U S A. 2009;106(20):8320-5. 
18. Ferreira C, Palmer D, Blake K, Garden OA, Dyson J. Reduced Regulatory T Cell Diversity in NOD Mice Is Linked to Early Events in the Thymus. J Immunol. 2014;192(9):4145-52.

19. Wicker LS, Todd JA, Peterson LB. Genetic control of autoimmune diabetes in the NOD mouse. Annu Rev Immunol. 1995;13:179-200.

20. Thiault N, Darrigues J, Adoue VV, Gros M, Binet BB, Perals C, et al. Peripheral regulatory T lymphocytes recirculating to the thymus suppress the development of their precursors. Nat Immunol. 2015;16(6):628-34.

21. Darrigues J, Santamaria JC, Galindo-Albarrán A, Robey EA, Joffre OP, van Meerwijk JPM, et al. Robust intrathymic development of regulatory T cells in young NOD mice is rapidly restrained by recirculating cells. Eur J Immunol. 2021;51(3):580-93.

22. Fontenot JD, Dooley JL, Farr AG, Rudensky AY. Developmental regulation of Foxp3 expression during ontogeny. J Exp Med. 2005;202(7):901-6.

23. Yu W, Nagaoka H, Jankovic M, Misulovin Z, Suh H, Rolink A, et al. Continued RAG expression in late stages of B cell development and no apparent re-induction after immunization. Nature. 1999;400(6745):682-7.

24. Borgulya P, Kishi H, Uematsu Y, von Boehmer H. Exclusion and inclusion of alpha and beta T cell receptor alleles. Cell. 1992;69(3):529-37.

25. McCaughtry TM, Wilken MS, Hogquist KA. Thymic emigration revisited. J Exp Med. 2007;204(11):2513-20.

26. Romagnoli P, Dooley J, Enault G, Vicente R, Malissen B, Liston A, et al. The thymic niche does not limit development of the naturally diverse population of mouse regulatory T lymphocytes. J Immunol. 2012;189(8):3831-7.

27. Viret C, Leung-Theung-Long S, Serre L, Lamare C, Vignali DAA, Malissen B, et al. 
Thymus-specific serine protease controls autoreactive CD4 T cell development and autoimmune diabetes in mice. J Clin Invest. 2011;121(5):1810-21.

28. Feng $\mathrm{Y}$, van der Veeken J, Shugay M, Putintseva E V, Osmanbeyoglu HU, Dikiy S, et al. A mechanism for expansion of regulatory $\mathrm{T}$-cell repertoire and its role in selftolerance. Nature. 2015;528(7580):132-6.

29. Britanova O V., Putintseva E V., Shugay M, Merzlyak EM, Turchaninova MA, Staroverov DB, et al. Age-Related Decrease in TCR Repertoire Diversity Measured with Deep and Normalized Sequence Profiling. J Immunol. 2014;192(6):2689-98.

30. Vander Heiden JA, Yaari G, Uduman M, Stern JNH, O'connor KC, Hafler DA, et al. PRESTO: A toolkit for processing high-throughput sequencing raw reads of lymphocyte receptor repertoires. Bioinformatics. 2014;30(13).

31. Bolotin DA, Poslavsky S, Mitrophanov I, Shugay M, Mamedov IZ, Putintseva E V., et al. MiXCR: Software for comprehensive adaptive immunity profiling. Nat Methods. 2015;12(5):380-1.

32. Shugay M, Bagaev D V., Turchaninova MA, Bolotin DA, Britanova O V., Putintseva E V., et al. VDJtools: Unifying Post-analysis of T Cell Receptor Repertoires. PLoS Comput Biol. 2015;11(11):1-16.

33. Haegeman B, Hamelin J, Moriarty J, Neal P, Dushoff J, Weitz JS. Robust estimation of microbial diversity in theory and in practice. ISME J. 2013;7(6):1092-101.

34. Matsutani T, Ogata M, Fujii Y, Kitaura K, Nishimoto N, Suzuki R, et al. Shortening of complementarity determining region 3 of the T cell receptor $\alpha$ chain during thymocyte development. Mol Immunol. 2011;48(4):623-9.

35. Yassai M, Gorski J. Thymocyte Maturation: Selection for In-Frame TCR $\alpha$-Chain Rearrangement Is Followed by Selection for Shorter TCR $\beta$-Chain Complementarity- 
Determining Region 3. J Immunol. 2000;165(7):3706-12.

36. Yassai M, Ammon K, Goverman J, Marrack P, Naumov Y, Gorski J. A Molecular Marker for Thymocyte-Positive Selection: Selection of CD4 Single-Positive Thymocytes with Shorter TCRB CDR3 During T Cell Development. J Immunol. 2002;168(8):3801-7.

37. Matsutani T, Ohmori T, Ogata M, Soga H, Kasahara S, Yoshioka T, et al. Comparison of CDR3 length among thymocyte subpopulations: Impacts of MHC and BV segment on the CDR3 shortening. Mol Immunol. 2007;44(9):2378-87.

38. Gilfillan S, Waltzinger C, Benoist C, Mathis D. More efficient positive selection of thymocytes in mice lacking terminal deoxynucleotidyl transferase. Int Immunol. 1994;6(11):1681-6.

39. Luther SA, Acha-Orbea H. Mouse mammary tumor virus: immunological interplays between virus and host. Adv Immunol. 1997;65:139-243.

40. Lio CWJ, Hsieh CS. A Two-Step Process for Thymic Regulatory T Cell Development. Immunity. 2008;28(1):100-11.

41. Fisson S, Darrasse-Jeze G, Litvinova E, Septier F, Klatzmann D, Liblau R, et al. Continuous Activation of Autoreactive CD4+ CD25+ Regulatory T Cells in the Steady State. J Exp Med. 2003;198(5):737-46.

42. Venanzi ES, Melamed R, Mathis D, Benoist C. The variable immunological self: Genetic variation and nongenetic noise in Aire-regulated transcription. Proc Natl Acad Sci U S A. $2008 ; 105(41): 15860-5$. 


\section{Legends to the figures}

Figure 1

The TCR-V $\beta$ repertoires expressed by Treg newly developed in NOD vs. B6 thymi are similarly diverse

(A) Thymocytes from eight-week-old Rag2-Gfp Foxp3-Thy $1^{a}$ mutant NOD females were analyzed by flow-cytometry using antibodies to indicated markers labeled with indicated fluorochromes. Gating strategy used to sort and quantify newly developed (i.e. GFP+) Treg (left panels). Proportions of newly developed $\left(\mathrm{GFP}^{+}\right)$and recirculating $\left(\mathrm{GFP}^{-}\right)$cells among thymic Treg (right panels). $n=14$, four independent experiments. (B) Thymocytes from eightweek-old wt B6 and NOD females were analyzed by flow-cytometry using antibodies to CD4, CD8, CD73, CCR7, Foxp3, and a panel of antibodies to indicated TCR-V $\beta$. Depicted are the mean distributions of TCR-V $\beta$ usage among newly developed $C D 4^{+} C D 8^{-}$ $\mathrm{CCR}^{\text {high }} \mathrm{CD}^{-} 3^{-/ \text {low }}$ Foxp3 ${ }^{+}$Treg in indicated mouse strains (see Fig. S1 for CCR7/CD73-gates). The indicated diversity-measure $D$ is the Shannon-diversity (i.e. the exponential of the Shannon entropy, see Research designs and methods section), represented as mean \pm SD ( $\mathrm{n}=6$ mice per strain). ${ }^{*} P<0.05$ (Mann-Whitney test). In Fig. S2A, data for individual TCR-V $\beta$ are depicted. 
Figure 2

Similar V and J segment usage and CDR3-lengths of TCR $\alpha$ and TCR $\beta$ expressed by Treg newly developed in NOD vs. B6 thymi

$\mathrm{CD}^{+} \mathrm{CD} 8{ }^{-}$Thy $1.1^{+} \mathrm{GFP}^{+}$Treg newly developed in the thymus of eight-week-old Rag2-Gfp Foxp3-Thy $1^{a}$ mutant B6 and NOD female mice were FACS-sorted and analyzed by UMIbased TCRseq. (A) TRAV-, (B) TRBV-, (C) TRAV/TRAJ, and (D) TRBV/TRBJ-usage, mean values of the three samples. These graphs summarize the data listed in Tables S1-S4. In (C) and (D) $\mathrm{V}$ and $\mathrm{J}$ segments are ordered as in the genome (arrows). Measures of position-dependent usages of TRAV and TRAJ are shown in Fig. S4. The indicated diversity-measure " $D$ " is the Shannon-diversity (i.e. the exponential of the Shannon entropy), represented as mean \pm SD. CDR3 $\alpha(E)$ and CDR3 $\beta(F)$ lengths in amino-acids. CDR3s starts with conserved Cys and Ala and end with conserved Phe. $(\mathrm{G}, \mathrm{H})$ Number of (non-germline-encoded) N-nucleotides and $(\mathrm{I}, \mathrm{J})$ (palindromic) P-nucleotides in the $\operatorname{CDR} 3 \alpha(\mathrm{G}, \mathrm{I})$ and $\operatorname{CDR} 3 \beta(\mathrm{H}, \mathrm{J})$. In E-J, left panels indicate mean values $\pm S D$, left panels distribution (mean values $\pm S D$ ). $n=3$ pools of eight to ten thymi per strain. $\mathrm{ns}=$ not significant, $* P<0.05$ (Mann-Whitney test). 
Figure 3

Similar diversities of TCR $\alpha$ and TCR $\beta$ clonotypes expressed by Treg newly developed in NOD vs. B6 thymi

$\mathrm{CD}^{+} \mathrm{CD} 8$-Thy1.1 $1^{+} \mathrm{GFP}^{+}$Treg newly developed in the thymi of eight-week-old Rag2-Gfp Foxp3Thy $1^{a}$ mutant B6 and NOD female mice were FACS-sorted and analyzed by UMI-based TCRseq. (A) Rarefaction plots of data pooled from the three experiments for TCR $\alpha$ (left) and TCR $\beta$ (right) clonotypes (as determined by V-usage, CDR3-sequence, and J-usage) in the indicated strains. For rarefaction-plots of individual experiments, see Fig. S5A. Shadows indicate $95 \%$ sampling-confidence intervals. (B) Shannon diversities (i.e. the exponential of the Shannon entropy, top) and Chao1 estimates (bottom) for TCR $\alpha$ (left) and TCR $\beta$ (right)clonotypes. $n s=$ not significant (Mann-Whitney test). Bars indicate mean values + SD. (C) Mean clonotype frequencies for TCR $\alpha$ using TRAV2 or TRAV17 in indicated strains. The diversity measure $D$ indicates Shannon-diversity \pm SD. (D) Rarefaction plots of pooled data for TCR $\alpha$ using TRAV2 or TRAV17 in indicated strains. Shadows indicate 95\% samplingconfidence intervals. For rarefaction-plots of individual experiments, see Fig. S5C. $n=3$ pools of eight to ten thymi per strain. 
Figure 1

A

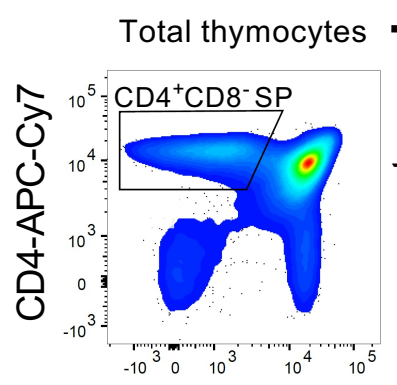

CD8-PECF594

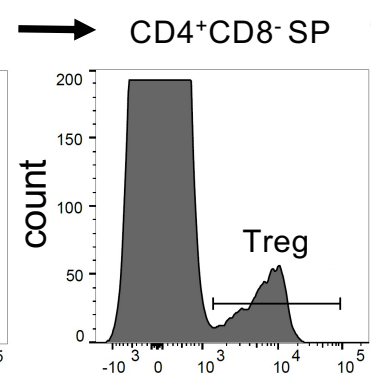

Thy1.1-APC

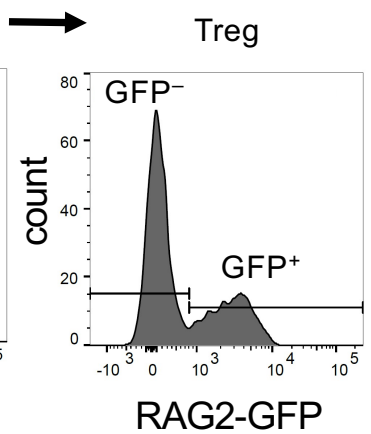

RAG2-GFP

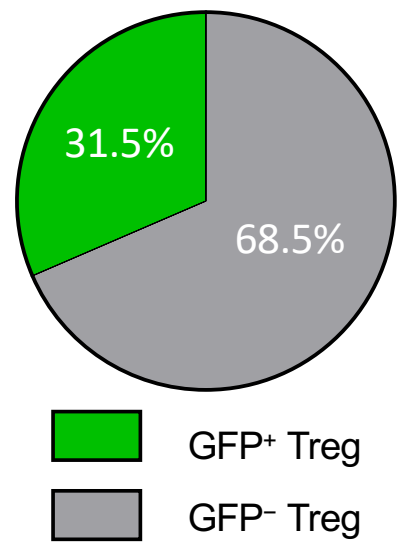

B
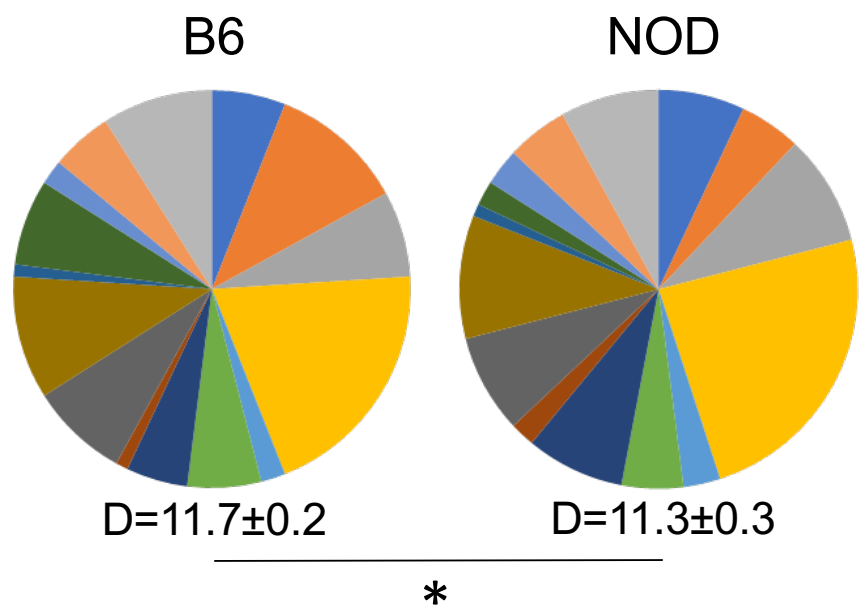

$\mathrm{V} \beta 2$

$\vee \beta 5.1 / 5.2$

V $\beta 8.3$

V $\beta 8.1 / 8.2$

$\vee \beta 13$

V $\beta 12$

V $\beta 11$

V $\beta 9$
V $\beta 6$

V $\beta 4$

- $\vee \beta 17 a$

- $\vee \beta 3$

- $\vee \beta 7$

V $\beta 10 b$

V $\beta 14$

$$
D=11.7 \pm 0.2
$$

* 


\section{Figure 2}

A

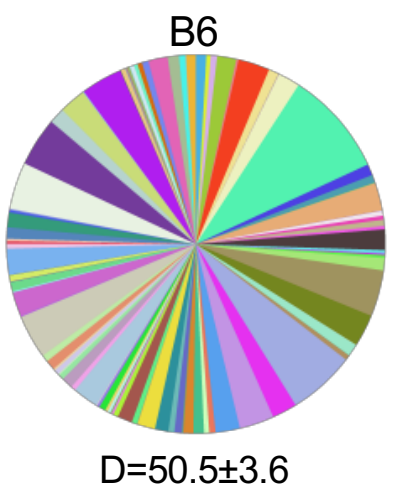

$D=50.5 \pm 3.6$

C

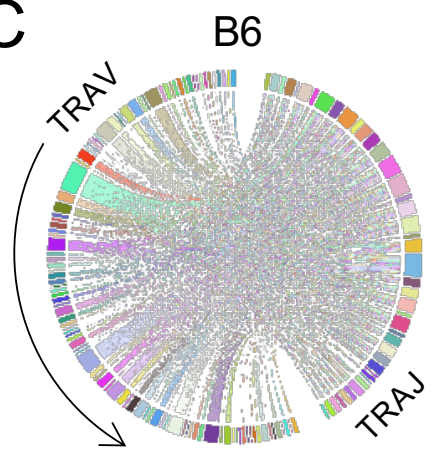

$D=1172.9 \pm 124.8$

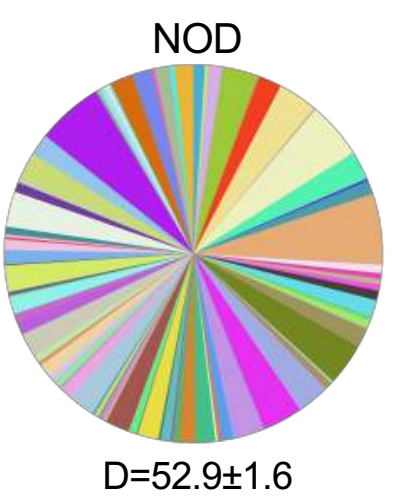

ns

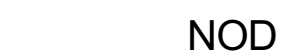

B

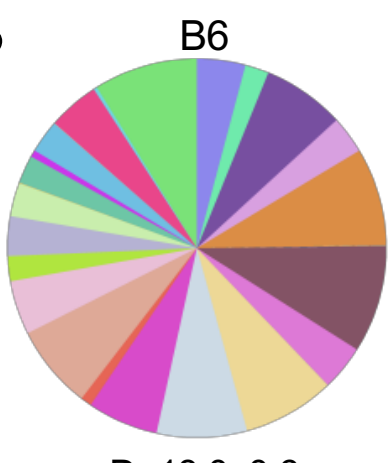

$D=18.0 \pm 0.6$

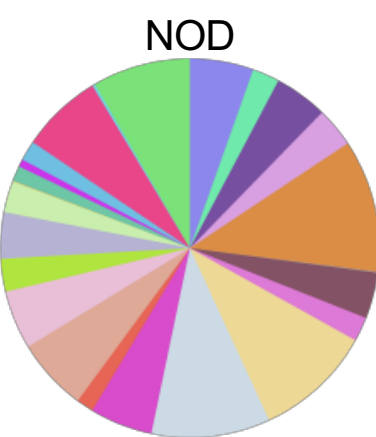

$D=17.3 \pm 1.1$

ns

D

B6

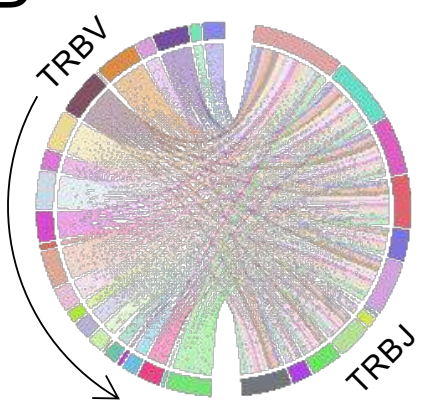

$D=171.0 \pm 4.2$

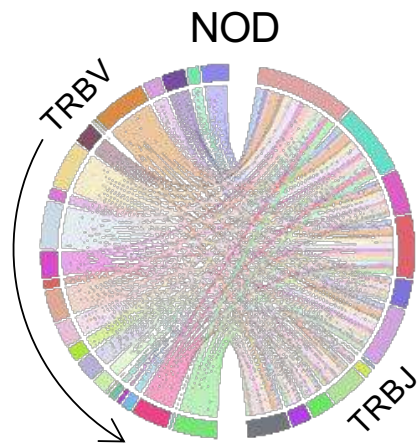

$D=158.7 \pm 7.4$

ns
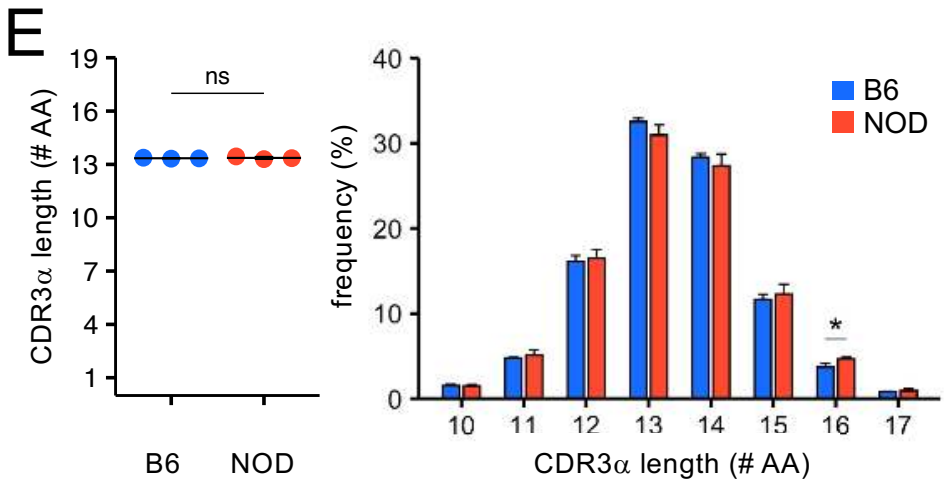

E
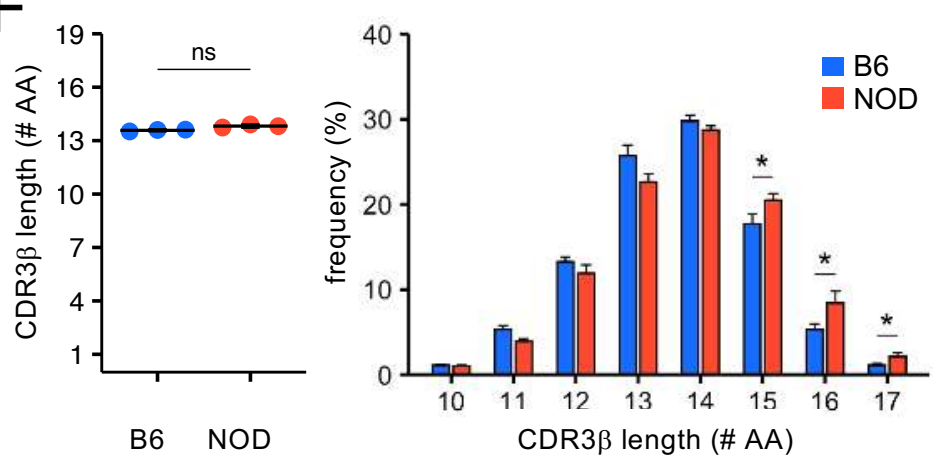

$\mathrm{H}$
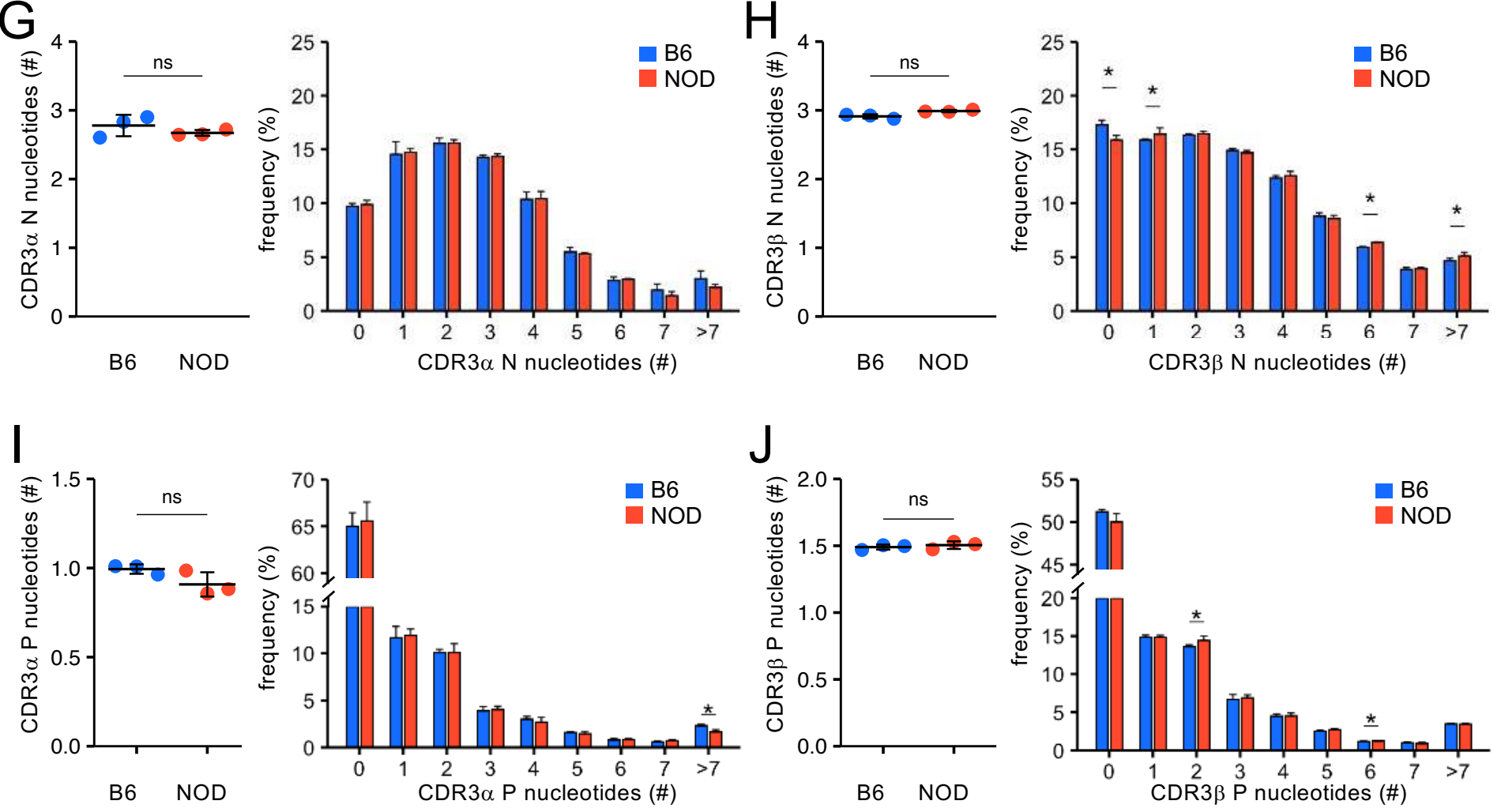
Figure 3

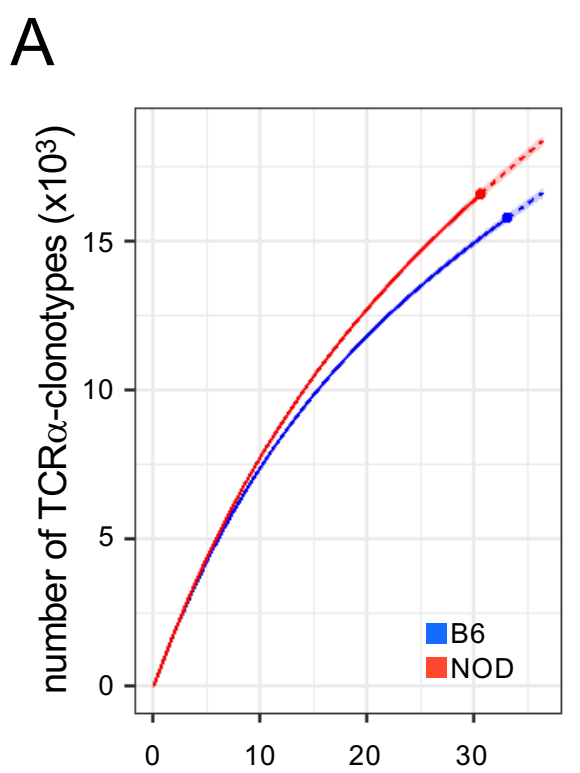

TCR $\alpha$ sample-size $\left(x 10^{3}\right)$
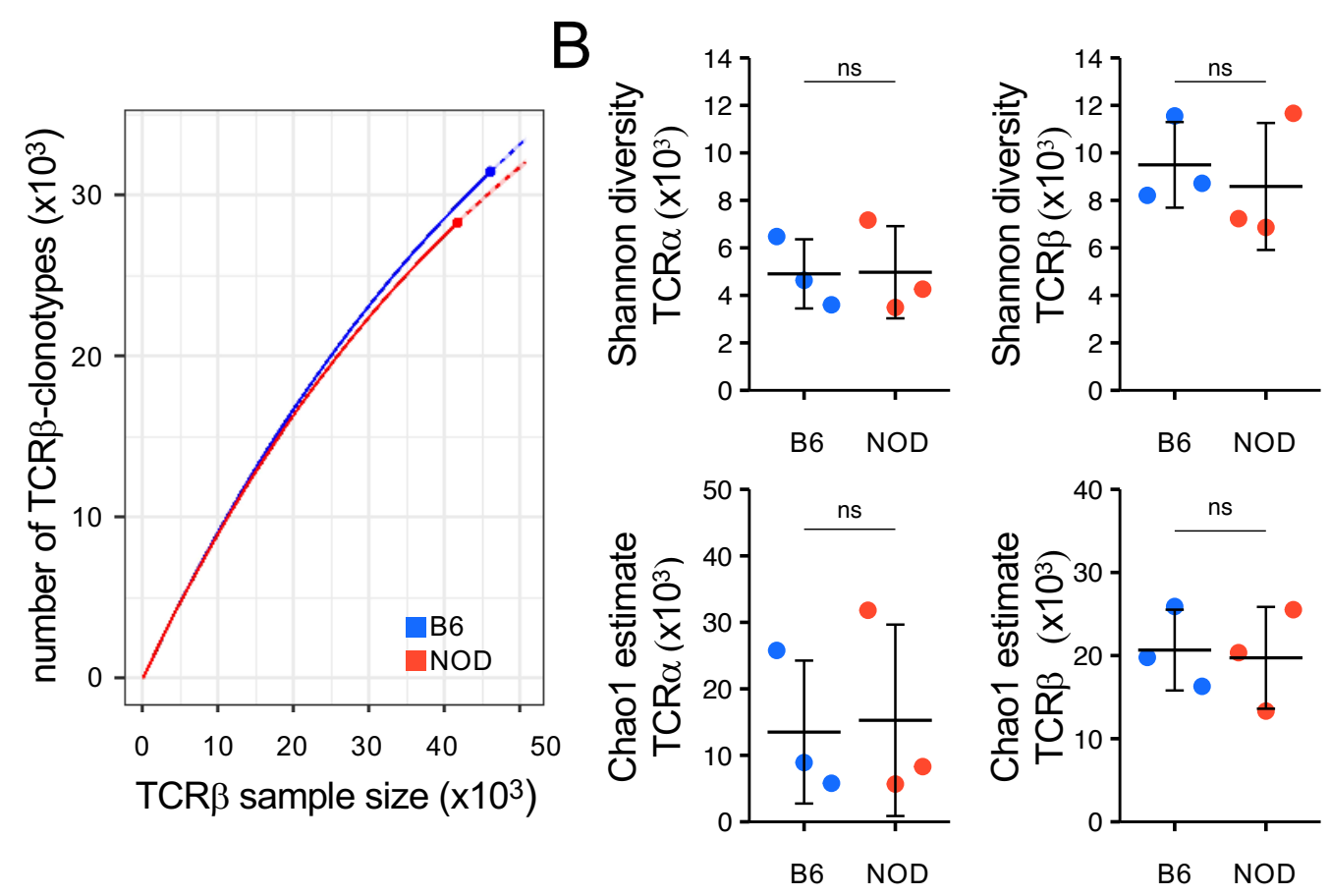

C

B6

NOD

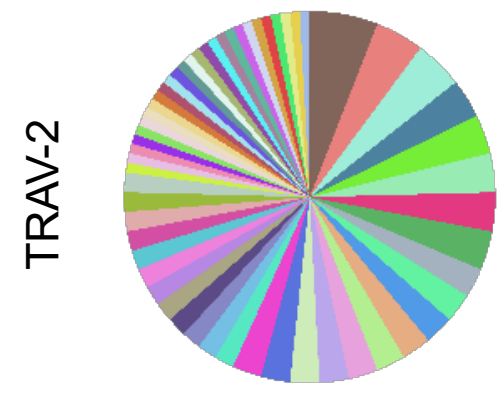

$D=18.2 \pm 3.6$

$D=14.3 \pm 8.2$

ns

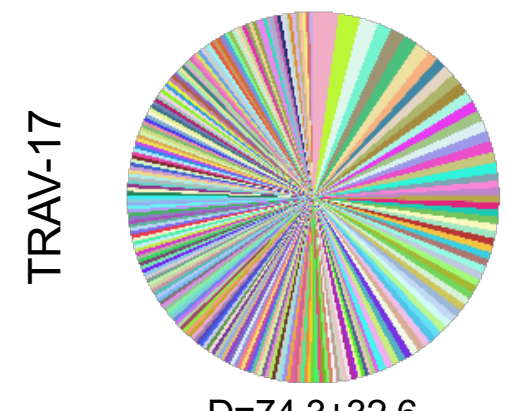

$D=74.3 \pm 32.6$
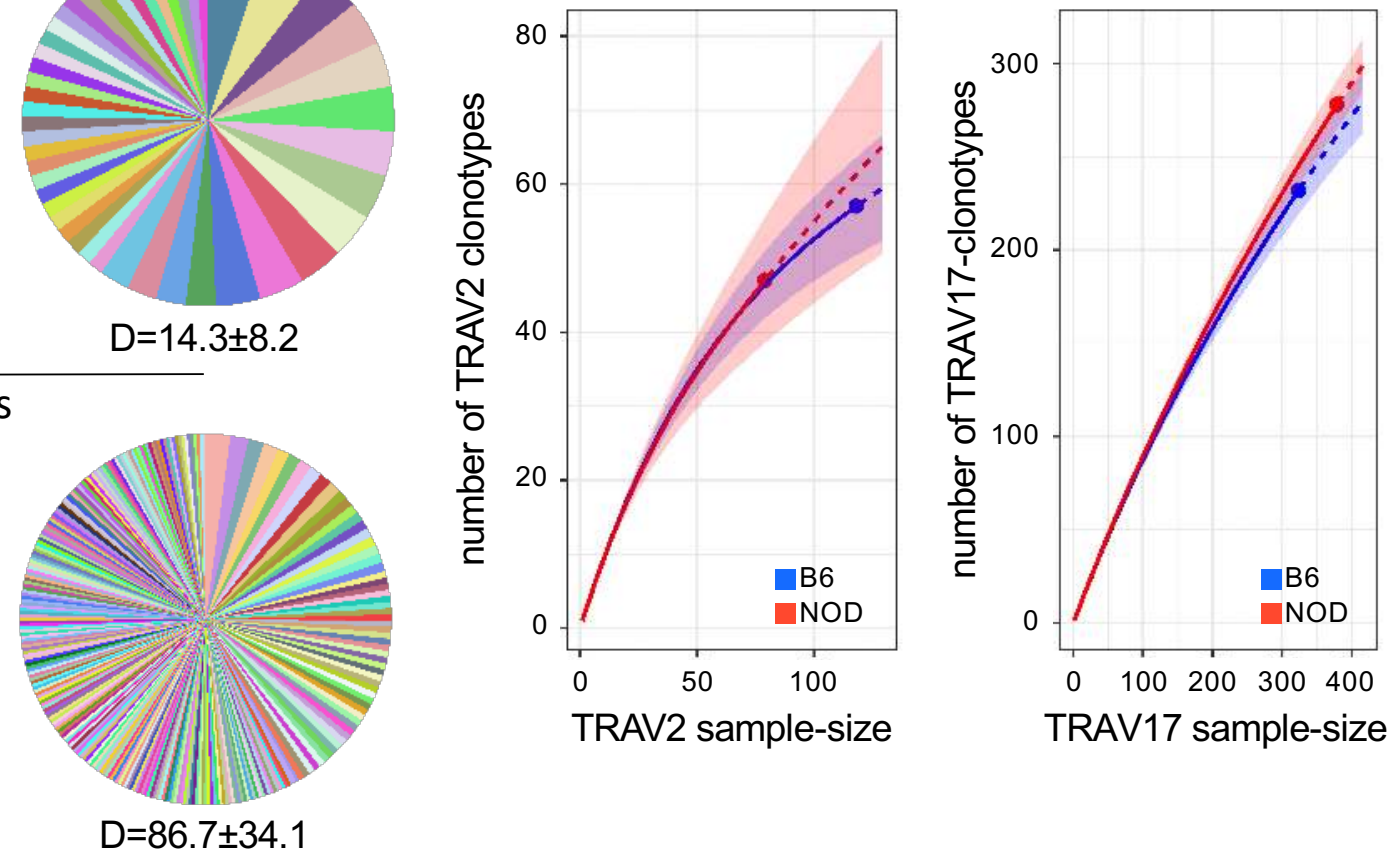

ns 